\title{
Prevalence of Intestinal Protozoa among Saudi Patients with Chronic Renal Failure: A Case-Control Study
}

\author{
Yousry A. Hawash, ${ }^{1,2}$ Laila Sh. Dorgham, ${ }^{3}$ El-Amir M. Amir, ${ }^{2,4}$ and Osama F. Sharaf ${ }^{2}$ \\ ${ }^{1}$ Department of Medical Laboratory Science, College of Applied Medical Sciences, Taif University, Taif 21944, Saudi Arabia \\ ${ }^{2}$ Department of Medical Parasitology, National Liver Institute, Menoufia University, Shebin El-Koom, Menoufia 23513, Egypt \\ ${ }^{3}$ Department of Community Medicine, National Liver Institute, Menoufia University, Shebin El-Koom, Menoufia 23513, Egypt \\ ${ }^{4}$ Parasitology Department, Rabigh Medical College, King Abdulaziz University, Jeddah 21589, Saudi Arabia
}

Correspondence should be addressed to Yousry A. Hawash; yousryhawash@gmail.com

Received 18 June 2015; Revised 3 September 2015; Accepted 8 September 2015

Academic Editor: Carlos E. P. Corbett

Copyright (C) 2015 Yousry A. Hawash et al. This is an open access article distributed under the Creative Commons Attribution License, which permits unrestricted use, distribution, and reproduction in any medium, provided the original work is properly cited.

\begin{abstract}
It has been hypothesized that chronic renal failure (CRF) predisposes patients to infection with intestinal protozoa. We tested this hypothesis with a matched case-control study to determine the prevalence of these protozoa and their diarrhea associated symptoms among 50 patients with CRF (cases) from Taif, western Saudi Arabia. Fifty diarrheal patients without CRF were recruited in the study as controls. Participants were interviewed by a structured questionnaire and stool samples were collected. Samples were thoroughly examined with microscopy and three coproantigens detection kits. Enteric protozoa were detected in 21 cases and 14 controls. Blastocystis spp. were the most predominant parasite (16\% in cases versus $8 \%$ in controls), followed by Giardia duodenalis (10\% in cases versus $12 \%$ in controls) and Cryptosporidium spp. (10\% in cases versus $6 \%$ in controls). Cyclospora cayetanensis was identified in two cases, while Entamoeba histolytica was described in one case and one control. Intestinal parasitism was positively associated with the male gender, urban residence, and travel history. Clinical symptoms of nausea/vomiting and abdominal pain were significantly varied between the parasitized cases and controls $(P$ value $\leq 0.05)$. Given the results, we recommend screening all diarrheal feces for intestinal protozoa in the study's population, particularly those with CRF.
\end{abstract}

\section{Introduction}

Enteric protozoa are a diverse group of unicellular microparasites inhabiting the intestinal tract of high vertebrate hosts including humans [1]. Infections usually occur through ingestion of cysts/oocysts contaminating raw food or drinking water $[2,3]$. Diarrhea is relatively a frequent symptom for protozoan infections but asymptomatic colonization is also common [1]. Attributing diarrhea to an exact parasite identified in a patient's feces is not certain for all protozoa. While a number of intestinal protozoa such as Giardia duodenalis, Entamoeba histolytica, Cryptosporidium spp., Cyclospora cayetanensis, Cystoisospora belli, and Microsporidia spp. have been certainly recognized to cause diarrhea in humans [4], others like Entamoeba coli and Entamoeba dispar almost certainly have not [5]. In addition, enteric protozoa like Blastocystis spp. and Dientamoeba fragilis have been recently identified in patients with diarrhea, but their causal role is still uncertain [6]. Regardless of the underlying protozoan parasite, diarrhea is usually mild and self-limited in healthy immunocompetent persons. Nevertheless, severe and protracted diarrhea has been reported in immunosuppressed patients [7].

By definition, patient with chronic renal failure (CRF) is a patient with end stage kidney disease causing marked decline in the glomerular filtration rate as well as uremia and requires kidney replacement or scheduled dialysis to survive [8]. It has been hypothesized that patients, at this stage, are more susceptible to infectious diarrhea than the normal population, secondary to immunosuppression $[9,10]$. This infectious diarrhea has been found associated with a number of intestinal viruses, bacteria, and protozoa [11]. In Saudi Arabia, high prevalence of enteric protozoa-related diarrhea has been reported [12] and the number of patients with CRF has been found rising [13]. Nevertheless, the frequency of these 
protozoa among patients with CRF has been poorly studied. In a case-control study, we tested the above hypothesis using a matched population from Taif, western Saudi Arabia. The burden of protozoan infections, associated symptoms, and potential risk factors were also sought in this study.

\section{Materials and Methods}

2.1. Study Site and Population. Two hundred and four patients comprising 139 with CRF and 64 without CRF from those patients attending the outpatient clinics at King Abdulaziz Specialized Hospital, Taif, Saudi Arabia, seeking treatment of their diarrheal episodes, were invited to participate in the study.

2.2. Exclusion Criteria. An episode of diarrhea was defined as $\geq 3$ loose bowel movements over a day prior to interviewing. Individuals with history of diabetes mellitus, malignancy, autoimmune disease, or any other chronic diseases were excluded from the study. Patients with history of taking antiparasitic, antidiarrheal, or immunosuppressive medications, 2 weeks prior to meeting, were also excluded from participation in the study. Following these exclusion criteria, 50 patients with CRF, described as cases, and 50 patients without CRF, named as controls, were allocated in the current study.

2.3. Ethical Consideration. All participants were informed of the purpose of our study and signed consent forms authorizing their voluntary participation. The regional ethics committee approved data collection, clinical samples collection, and analysis of the study.

2.4. Interviews and Questionnaire. On enrollments, face-toface interviews were conducted using a structured questionnaire for the basic demographic data in terms of age, sex, and residence. Questions about drinking water resources, history of travel to foreign countries, and/or exposure to animals during the three-week period preceding diarrhea were also included. Moreover, some clinical data concerning the number of bowels/day, duration of diarrhea, accompanying nausea/vomiting, mucous or blood in feces, abdominal pain, bloating/flatulence, general fatigue, and loss of weight were also sought for in the questionnaire.

2.5. Fecal Samples Collection. A total of 100 fecal samples, one sample from each participant, were collected between June and December 2014. Fresh samples were obtained in clean screw-capped cups. Feces were transported to the Microbiology Laboratory at College of Applied Medical Sciences, Taif University, within 2-3 hours after collection. Samples were appropriately coded and immediately stored at $4^{\circ} \mathrm{C}$ till the time of parasitological examination.

2.6. Parasitological Examination. Fresh feces were examined for consistency and presence of mucous or blood. Direct wet mount smears were microscopically examined for parasites ova, cysts, oocysts, and/or larvae, as previously described [14]. Formol-ether fecal concentrates of each sample were individually smeared and stained with native iodine, trichrome, modified trichrome, and modified Ziehl-Neelsen stains for identification of Blastocystis spp., Microsporidium spp., and intestinal coccidian protozoa, respectively. Staining and examination techniques were carried out according to Garcia, 2009 [15].

Fresh fecal specimens were also examined for G. duodenalis with RIDA Quick Giardia (R-Biopharm, Darmstadt, Germany), Cryptosporidium spp. with RIDA Quick Cryptosporidium (R-Biopharm, Darmstadt, Germany), and for E. histolytica with E. histolytica II Test (TechLab, Blacksburg, Virginia, USA) commercial kits. Immunoassays were performed following the corresponding manufacturer's protocol.

2.7. Statistical Methods. Statistical package for social science (SPSS) program, version 16 for windows, was used for data entry and data analysis. Summaries with descriptive statistics were generated and the data was further statistically analyzed according to the objectives of the study. Appropriate statistical tests (parametric or nonparametric tests) were used according to the type of data whether qualitative or quantitative. $P$ value $\leq 0.05$ was considered significant.

\section{Results}

3.1. Demographic Characteristics. Among 100 participants, 67 were males (mean age of $54.4 \pm 12.8$ ) and 33 were females (mean age of $51.9 \pm 14.3$ ). Eighty-three participants were urban, while the remaining 13 participants were from remote rural areas. Forty-seven patients had travel history to one tropical country 1-3 months before their diarrheal episodes. Twenty-six participants had contact with one or more domestic animal 3-4 weeks prior to their diarrheal episodes. Treated bottled water was the main drinking water resource for 56 participants, followed by desalted sea water for 31 participants and the underground well water for 13 participants.

3.2. Parasitic Infections. Intestinal protozoa were detected in $21(42 \%)$ cases and $14(28 \%)$ controls, with an overall prevalence rate of $35 \%$. No significant differences were statistically observed between both groups $(P=0.14)$. Blastocystis spp. were the most frequently identified parasite $(12 / 100 ; 12 \%)$ followed by $G$. duodenalis $(11 / 100 ; 11 \%)$ and Cryptosporidium spp. (8/100; 8\%). Blastocystis spp., Cryptosporidium spp., G. duodenalis, C. cayetanensis, and E. histolytica were identified in cases at infection rates of $16 \%(8 / 50), 10 \%(5 / 50), 10 \%$ $(5 / 50), 4 \%(2 / 50)$, and $2 \%(1 / 50)$, respectively. In the control group, Blastocystis spp., Cryptosporidium spp., G. duodenalis, and $E$. histolytica were diagnosed with prevalence rates of $8 \%(4 / 50), 6 \%(3 / 50), 12 \%(6 / 50)$, and $2 \%(1 / 50)$, respectively (Figure 1). None of these infections showed significant statistical difference between the two groups.

Concomitant parasitic infections were described in 5 cases $(5 / 100 ; 5 \%)$ and were absent in the control group. Cryptosporidium spp. were concurrently identified with $G$. duodenalis in a case and with C. cayetanensis in another. Blastocystis 
TABLE 1: The demographic characteristics variations among the parasitized and nonparasitized diarrheal cases and controls.

\begin{tabular}{|c|c|c|c|c|c|c|c|c|c|c|}
\hline \multirow{3}{*}{ Demographic character } & \multicolumn{5}{|c|}{ Protozoa-positive } & \multicolumn{5}{|c|}{ Protozoa-negative } \\
\hline & \multicolumn{2}{|c|}{ Cases } & \multicolumn{2}{|c|}{ Controls } & \multirow{2}{*}{$P$ value } & \multicolumn{2}{|c|}{ Cases } & \multicolumn{2}{|c|}{ Controls } & \multirow{2}{*}{$P$ value } \\
\hline & $n$ & $\%$ & $n$ & $\%$ & & $n$ & $\%$ & $n$ & $\%$ & \\
\hline \multicolumn{11}{|l|}{ Age } \\
\hline Mean \pm SD & \multicolumn{2}{|c|}{$54.4 \pm 12.8$} & \multicolumn{2}{|c|}{$48.8 \pm 12.6$} & $0.2(\mathrm{NS})$ & \multicolumn{2}{|c|}{$48.6 \pm 13.2$} & \multicolumn{2}{|c|}{$51.9 \pm 14.3$} & 0.33 (NS) \\
\hline \multicolumn{11}{|l|}{ Gender } \\
\hline Male & 14 & $66.7 \%$ & 9 & $64.3 \%$ & \multirow{2}{*}{0.88 (NS) } & 21 & $72.4 \%$ & 23 & $63.9 \%$ & \multirow{2}{*}{0.46 (NS) } \\
\hline Female & 7 & $33.3 \%$ & 5 & $35.7 \%$ & & 8 & $27.6 \%$ & 13 & $36.1 \%$ & \\
\hline \multicolumn{11}{|l|}{ Residence } \\
\hline Urban & 16 & $76.2 \%$ & 9 & $64.3 \%$ & \multirow{2}{*}{0.47 (NS) } & 27 & $93.1 \%$ & 31 & $86.1 \%$ & \multirow{2}{*}{0.44 (NS) } \\
\hline Rural & 5 & $23.8 \%$ & 5 & $35.7 \%$ & & 2 & $6.9 \%$ & 5 & $13.9 \%$ & \\
\hline \multicolumn{11}{|l|}{ Travel history } \\
\hline Yes & 11 & $52.4 \%$ & 7 & $50 \%$ & \multirow{2}{*}{0.89 (NS) } & 14 & $48.3 \%$ & 15 & $41.7 \%$ & \multirow{2}{*}{0.59 (NS) } \\
\hline No & 10 & $47.6 \%$ & 7 & $50 \%$ & & 15 & $51.7 \%$ & 21 & $58.3 \%$ & \\
\hline \multicolumn{11}{|l|}{ Animal contact } \\
\hline Yes & 7 & $33.3 \%$ & 6 & $42.9 \%$ & \multirow{2}{*}{0.56 (NS) } & 7 & $24.1 \%$ & 6 & $16.7 \%$ & \multirow{2}{*}{0.45 (NS) } \\
\hline No & 14 & $66.7 \%$ & 8 & $57.1 \%$ & & 22 & $75.9 \%$ & 30 & $83.3 \%$ & \\
\hline \multicolumn{11}{|l|}{ Drinking water } \\
\hline Bottled & 11 & $52.4 \%$ & 6 & $42.9 \%$ & \multirow{4}{*}{0.83 (NS) } & 19 & $65.5 \%$ & 20 & $55.6 \%$ & \multirow{4}{*}{0.007 (Sig) } \\
\hline Desalted & 8 & $38.1 \%$ & 6 & $42.9 \%$ & & 3 & $10.3 \%$ & 14 & $38.9 \%$ & \\
\hline Well & 2 & $9.5 \%$ & 2 & $14.3 \%$ & & 7 & $24.1 \%$ & 2 & $5.6 \%$ & \\
\hline Total & 21 & $100 \%$ & 14 & $100 \%$ & & 29 & $100 \%$ & 36 & $100 \%$ & \\
\hline
\end{tabular}

SD: standard deviation, $n$ : number, NS: nonsignificant, and Sig: significant. $P<0.005$.

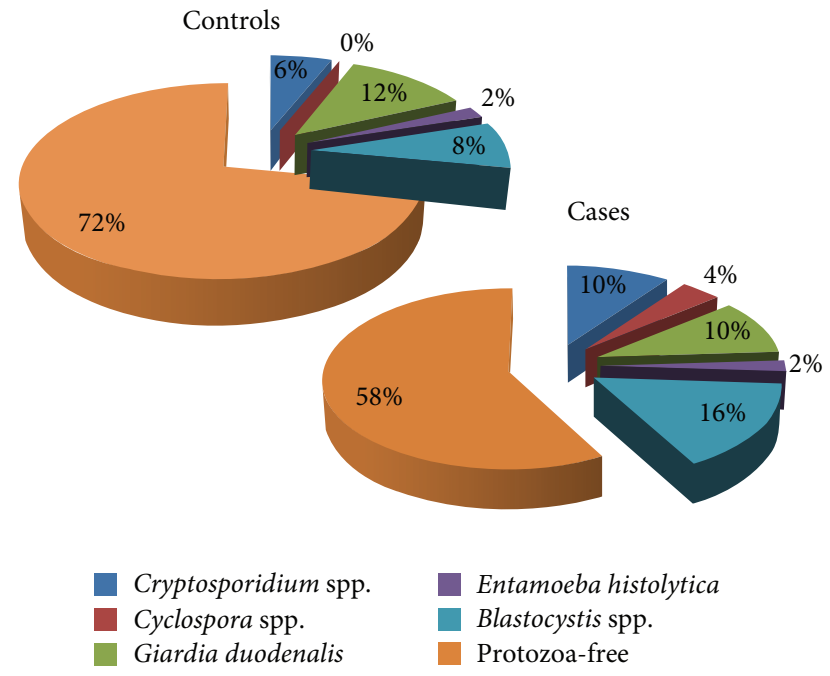

Figure 1: Parasitological tests results for 50 cases and 50 controls. Percentages of protozoan infection were written inside or outside color-matched pie charts slices for comparison.

spp. were concomitantly detected with E. histolytica in a case and with $G$. duodenalis in two cases. No helminths infection was observed for either cases or the control group. Neither C. belli nor Microsporidia spp. were detected in the study's participants. Importantly, 2 participants, one from each group, diagnosed as positives for E. histolytica/E. dispar by microscopy, were proved as E. histolytica positives by the ELISA test. Equally important, identification of protozoan cysts/oocysts in patients feces (Figure 2) was confirmed by the corresponding immunoassay.

3.3. Parasitism and Patients' Demographic Characteristics. Concerning the parasitized cases $(n=21), 14(66.7 \%)$ were males and $7(33.3 \%)$ were females. Infections were more frequent in cases from urban areas $(16 / 21 ; 76.2 \%)$ than those residing rural areas $(5 / 21 ; 23.8 \%)$. Eleven cases $(11 / 21 ; 52.4 \%)$ had travel history and $10(10 / 21 ; 47.6 \%)$ cases had not. Among the parasitized controls $(n=14)$, intestinal protozoa were identified more in males $(9 / 14 ; 64.3 \%)$ than in females $(5 / 14$; $35.7 \%)$, in persons from urban areas $(9 / 14 ; 64.3 \%)$ than those residing rural areas $(5 / 14 ; 35.7 \%)$, and in participants with travel history $(7 / 14 ; 50 \%)$ than those without $(7 / 14 ; 50 \%)$. No significant differences were statistically observed for any of these variables between the parasitized cases and controls (Table 1).

3.4. Parasitism and Patients' Clinical Characteristics. Acute and transient diarrhea was reported in $71 \%(15 / 21)$ of the parasitized cases and by all parasitized controls $(14 / 14 ; 100 \%)$, with no significant differences observed $(P=0.06)$ between both groups. Diarrhea continued for a duration of more than 2 weeks only in $28.6 \%(6 / 21)$ of protozoa-positive cases. Persistent diarrhea was reported in two G. duodenalis positive cases, two Cryptosporidium spp. positive cases, one Blastocystis spp. positive case, and one $C$. cayetanensis positive case. Mucous and blood were seen in feces of two cases $(9.5 \% ; 2 / 21)$ and one 
TABLE 2: The clinical characteristics variations among the parasitized and non-parasitized diarrheal cases and controls.

\begin{tabular}{|c|c|c|c|c|c|c|c|c|c|c|}
\hline \multirow{3}{*}{ Clinical character } & \multicolumn{5}{|c|}{ Protozoa-positive } & \multicolumn{5}{|c|}{ Protozoa-negative } \\
\hline & \multicolumn{2}{|c|}{ Cases } & \multicolumn{2}{|c|}{ Controls } & \multirow{2}{*}{$P$ value } & \multicolumn{2}{|c|}{ Cases } & \multicolumn{2}{|c|}{ Controls } & \multirow{2}{*}{$P$ value } \\
\hline & $n$ & $\%$ & $n$ & $\%$ & & $n$ & $\%$ & $n$ & $\%$ & \\
\hline \multicolumn{11}{|l|}{ Diarrhea } \\
\hline$<14$ days & 15 & $71.4 \%$ & 14 & $100 \%$ & \multirow{2}{*}{0.06 (NS) } & 25 & $86.2 \%$ & 36 & $100 \%$ & \multirow{2}{*}{0.03 (Sig) } \\
\hline$>14$ days & 6 & $28.6 \%$ & 0 & $0 \%$ & & 4 & $13.8 \%$ & 0 & $0 \%$ & \\
\hline \multicolumn{11}{|l|}{ Mucous/blood } \\
\hline Yes & 2 & $9.5 \%$ & 1 & $7.1 \%$ & \multirow{2}{*}{1.0 (NS) } & 4 & $13.8 \%$ & 2 & $5.6 \%$ & \multirow{2}{*}{0.39 (NS) } \\
\hline No & 19 & $90.5 \%$ & 13 & $92.9 \%$ & & 25 & $86.2 \%$ & 34 & $94.4 \%$ & \\
\hline \multicolumn{11}{|l|}{ Abdominal pain } \\
\hline Yes & 21 & $100 \%$ & 11 & $78.6 \%$ & \multirow{2}{*}{0.05 (Sig) } & 29 & $100 \%$ & 28 & $77.8 \%$ & \multirow{2}{*}{0.007 (Sig) } \\
\hline No & 0 & $0 \%$ & 3 & $21.4 \%$ & & 0 & $0 \%$ & 8 & $22.2 \%$ & \\
\hline \multicolumn{11}{|l|}{ Nausea/vomiting } \\
\hline Yes & 12 & $57.1 \%$ & 13 & $92.9 \%$ & \multirow{2}{*}{0.02 (Sig) } & 2 & $6.9 \%$ & 4 & $11.1 \%$ & \multirow{2}{*}{$0.68(\mathrm{NS})$} \\
\hline No & 9 & $42.9 \%$ & 1 & $7.1 \%$ & & 27 & $93.1 \%$ & 32 & $88.9 \%$ & \\
\hline \multicolumn{11}{|l|}{ Bloating/flatulence } \\
\hline Yes & 16 & $76.2 \%$ & 10 & $71.4 \%$ & \multirow{2}{*}{$1.0(\mathrm{NS})$} & 18 & $62.1 \%$ & 6 & $16.7 \%$ & \multirow{2}{*}{$0.001(\mathrm{HS})$} \\
\hline No & 5 & $23.8 \%$ & 4 & $28.6 \%$ & & 11 & $37.9 \%$ & 30 & $83.3 \%$ & \\
\hline \multicolumn{11}{|l|}{ Fatigue } \\
\hline Yes & 21 & $100 \%$ & 14 & $100 \%$ & \multirow{2}{*}{ 一 } & 29 & $100 \%$ & 4 & $11.1 \%$ & \multirow{2}{*}{0.001 (HS) } \\
\hline No & 0 & $0 \%$ & 0 & $0 \%$ & & 0 & $0 \%$ & 32 & $88.9 \%$ & \\
\hline \multicolumn{11}{|l|}{ Loss of weight } \\
\hline Yes & 2 & $9.5 \%$ & 2 & $14.3 \%$ & \multirow{3}{*}{$1.0(\mathrm{NS})$} & 4 & $13.8 \%$ & 4 & $11.1 \%$ & \multirow{3}{*}{0.74 (NS) } \\
\hline No & 19 & $90.5 \%$ & 12 & $85.7 \%$ & & 25 & $85.2 \%$ & 32 & $88.9 \%$ & \\
\hline Total & 21 & $100 \%$ & 14 & $100 \%$ & & 29 & $100 \%$ & 36 & $100 \%$ & \\
\hline
\end{tabular}

n: number, NS: nonsignificant, Sig: significant, and HS: highly significant. $P<0.005$.

control $(7.1 \% ; 1 / 14)$. One of these two cases was positive for $E$. histolytica and Blastocystis spp., while the other was positive for Blastocystis spp., as a sole infection. The only patient in the control group who reported mucous and blood in his feces was E. histolytica positive. Bloating/flatulence, abdominal pain, general fatigue, and nausea/vomiting were the common symptoms found associated with the diarrhea (Table 2).

Diarrhea associated with abdominal pain and fatigue sensation was reported by all the parasitized cases. Diarrhea associated with nausea/vomiting, bloating/flatulence sensation, and losing some weight was reported by $\approx 57 \%$ $(12 / 21), \approx 76 \%(16 / 21)$, and $9.5 \%(2 / 21)$ of the parasitized cases, respectively. Among the parasitized controls $(n=$ 14), diarrhea accompanied with symptoms of abdominal pain, nausea/vomiting, bloating/flatulence sensation, feeling fatigued, and losing weight was practiced by $\approx 79 \%(11 / 14)$, $93 \%(13 / 14), \approx 71 \%(10 / 14)$, and $100 \%(14 / 14)$ of patients, respectively. Significant differences were found between parasitized cases and parasitized controls regarding the association of their diarrhea with abdominal pain $(P=0.05)$ and with nausea/vomiting $(P=0.02)$. No significant difference between the parasitized cases and controls was observed for other symptoms.

3.5. Blastocystis Species Associated Diarrhea and Patients' Clinical Characteristics. Diarrhea was often mild $(87.5 \%$ of cases versus $100 \%$ of controls) and nondysenteric (87.5\% of cases versus $100 \%$ of controls). The commonly associated symptoms were abdominal pain, fatigue (100\% of cases and controls), nausea/vomiting (87.5\% of cases versus $100 \%$ of controls), flatulence/bloating (75\% of cases versus $25 \%$ of controls), and losing weight (12.5\% of cases versus $0 \%$ of controls). No significant difference was found between the parasitized cases and controls (Table 3 ).

3.6. Cryptosporidium Species Associated Diarrhea and Patients' Clinical Characteristics. Cryptosporidium species associated diarrhea was often mild ( $60 \%$ of cases versus $100 \%$ of controls) and nondysenteric in all parasitized patients. The commonly reported associated symptoms were fatigue (100\% of cases and controls), abdominal pain (100\% of cases versus $33.3 \%$ of controls), nausea/vomiting (60\% of cases versus $100 \%$ of controls), flatulence/bloating ( $80 \%$ of cases versus $100 \%$ of controls), and losing weight (12.5\% of cases and controls). Statistically, no significant difference was described for any of these symptoms between the parasitized patients in both groups (Table 4).

3.7. Giardia duodenalis Associated Diarrhea and Patients' Clinical Characteristics. As described in Table 5, G. duodenalis associated diarrhea was frequently mild (60\% of cases versus $100 \%$ of controls) and nondysenteric (100\% of cases and $83.3 \%$ of controls). The common associated symptoms 


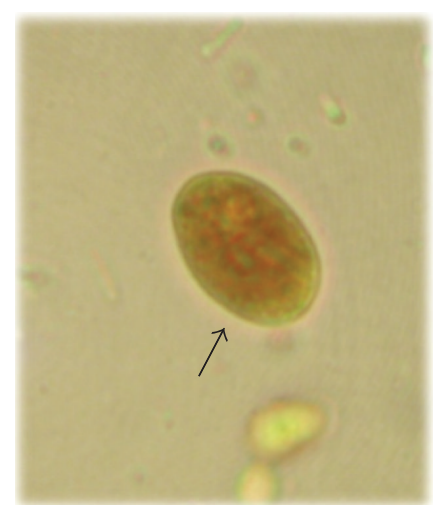

(a)

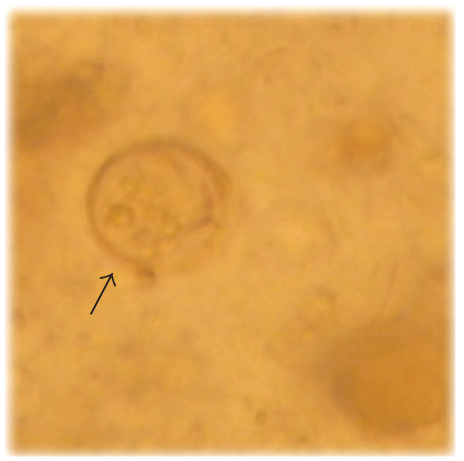

(c)

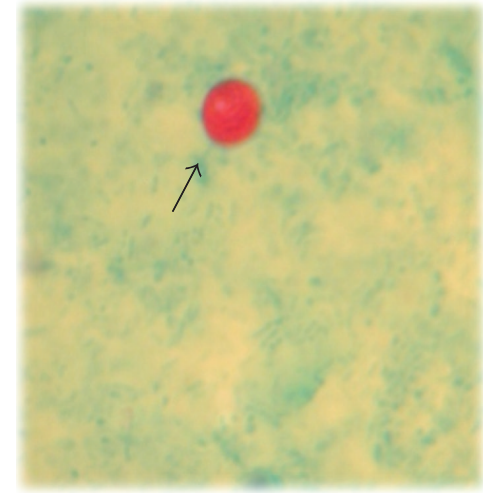

(b)

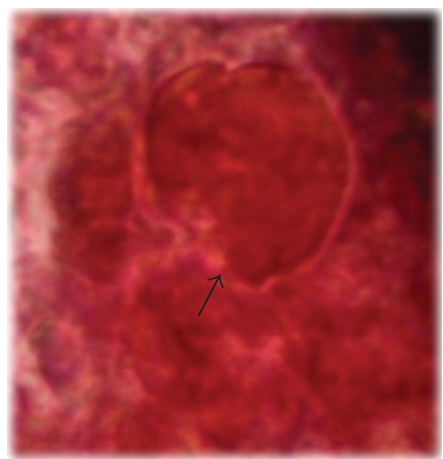

(d)

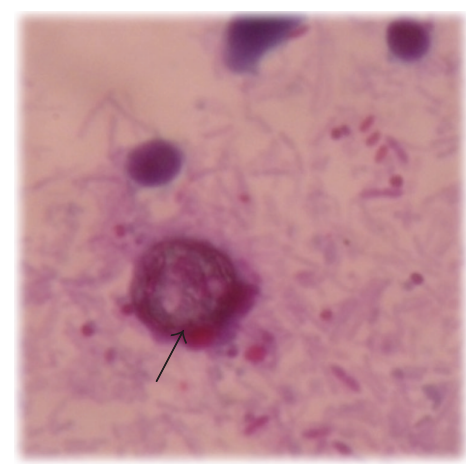

(e)

FIGURE 2: Merged microscopic pictures for wet mounts smears of formol-ether fecal concentrates showing the recognized enteric protozoa. (a) Giardia duodenalis cyst (iodine stain, 400x magnification), (b) Cryptosporidium spp. oocyst (modified Ziehl-Neelsen stain, 400x magnification), (c) Entamoeba histolytica/Entamoeba dispar cyst (iodine stain, 400x magnification), (d) Blastocystis spp. vacular form (trichrome stain, 1000x magnification), and (e) Cyclospora spp. oocyst (mZN stain, 400x magnification).

were fatigue (100\% of cases and controls), abdominal pain (100\% of cases versus $83.3 \%$ of controls), nausea/vomiting ( $80 \%$ of cases versus $83.3 \%$ of controls), flatulence/bloating complaints (100\% of cases versus $83.3 \%$ of controls), and losing weight ( $00 \%$ of cases versus $9 \%$ of controls). None of these symptoms showed significant statistical differences between the parasitized cases and controls.

\section{Discussion}

In the study's population, protozoan infections associated with diarrhea were common (35\%). Infections were identified in considerable number of patients in the diarrheal control group. This is not surprising for this population where several protozoan infections predominate [12]. Intestinal protozoa were identified more in cases than in control group, reflecting the high association between the isolated protozoa and diarrhea in patient with CRF. In agreement with this finding, in one case-control study carried out in Turkey, protozoan infections have been described in $\approx 44 \%(62 / 142)$ of cases and $\approx 13 \%(19 / 150)$ of controls [16]. In another study executed in Brazil, Kulik et al. have reported these infections in $\approx 45 \%$ (33/86) of cases and in $\approx 26 \%(36 / 146)$ of controls [17]. Contrary to our findings, Gil et al. have reported more protozoan 
TABLE 3: The clinical characteristics variations among Blastocystis species positive/negative diarrheal cases and controls.

\begin{tabular}{|c|c|c|c|c|c|c|c|c|c|c|}
\hline \multirow{3}{*}{ Clinical character } & \multicolumn{5}{|c|}{ Blastocystis species-positive } & \multicolumn{5}{|c|}{ Blastocystis species-negative } \\
\hline & \multicolumn{2}{|c|}{ Cases } & \multicolumn{2}{|c|}{ Controls } & \multirow{2}{*}{$P$ value } & \multicolumn{2}{|c|}{ Cases } & \multicolumn{2}{|c|}{ Controls } & \multirow{2}{*}{$P$ value } \\
\hline & $n$ & $\%$ & $n$ & $\%$ & & $n$ & $\%$ & $n$ & $\%$ & \\
\hline \multicolumn{11}{|l|}{ Diarrhea } \\
\hline$<14$ days & 7 & $87.5 \%$ & 4 & $100 \%$ & \multirow{2}{*}{$1.0(\mathrm{NS})$} & 33 & $78.6 \%$ & 46 & $100 \%$ & \multirow{2}{*}{0.001 (Sig } \\
\hline$>14$ days & 1 & $12.5 \%$ & 0 & $0 \%$ & & 9 & $21.4 \%$ & 0 & $0 \%$ & \\
\hline \multicolumn{11}{|l|}{ Mucous/blood } \\
\hline Yes & 1 & $12.5 \%$ & 0 & $0 \%$ & \multirow{2}{*}{1.0 (NS) } & 5 & $11.9 \%$ & 3 & $6.5 \%$ & \multirow{2}{*}{0.47 (NS) } \\
\hline No & 7 & $87.5 \%$ & 4 & $100 \%$ & & 37 & $88.1 \%$ & 43 & $93.5 \%$ & \\
\hline \multicolumn{11}{|l|}{ Abdominal pain } \\
\hline Yes & 8 & $100 \%$ & 4 & $100 \%$ & \multirow{2}{*}{ - } & 42 & $100 \%$ & 35 & $76.1 \%$ & \multirow{2}{*}{0.001 (Sig) } \\
\hline No & 0 & $0 \%$ & 0 & $0 \%$ & & 0 & $0 \%$ & 11 & $23.9 \%$ & \\
\hline \multicolumn{11}{|l|}{ Nausea/vomiting } \\
\hline Yes & 7 & $87.5 \%$ & 4 & $100 \%$ & \multirow{2}{*}{$1.0(\mathrm{NS})$} & 7 & $16.7 \%$ & 13 & $28.3 \%$ & \multirow{2}{*}{$0.2(\mathrm{NS})$} \\
\hline No & 1 & $12.5 \%$ & 0 & $0 \%$ & & 35 & $83.3 \%$ & 33 & $71.7 \%$ & \\
\hline \multicolumn{11}{|l|}{ Bloating/flatulence } \\
\hline Yes & 6 & $75 \%$ & 1 & $25 \%$ & \multirow{2}{*}{$0.22(\mathrm{NS})$} & 28 & $66.7 \%$ & 15 & $32.6 \%$ & \multirow{2}{*}{$0.003(\mathrm{Sig})$} \\
\hline No & 2 & $25 \%$ & 3 & $75 \%$ & & 14 & $33.3 \%$ & 34 & $67.4 \%$ & \\
\hline \multicolumn{11}{|l|}{ Fatigue } \\
\hline Yes & 8 & $100 \%$ & 4 & $100 \%$ & \multirow{2}{*}{ - } & 42 & $100 \%$ & 14 & $30.4 \%$ & \multirow{2}{*}{0.00 (HS) } \\
\hline No & 0 & $0 \%$ & 0 & $0 \%$ & & 0 & $0 \%$ & 32 & $69.6 \%$ & \\
\hline \multicolumn{11}{|l|}{ Loss of weight } \\
\hline Yes & 1 & $12.5 \%$ & 0 & $0 \%$ & \multirow{3}{*}{1.0 (NS) } & 5 & $11.9 \%$ & 6 & $13 \%$ & \multirow{3}{*}{$1.0(\mathrm{NS})$} \\
\hline No & 7 & $87.5 \%$ & 4 & $100 \%$ & & 37 & $88.1 \%$ & 40 & $87 \%$ & \\
\hline Total & 8 & $100 \%$ & 4 & $100 \%$ & & 42 & $100 \%$ & 46 & $100 \%$ & \\
\hline
\end{tabular}

n: number, NS: nonsignificant, Sig: significant, and HS: highly significant. $P<0.005$.

infections $(61 \%)$ in controls than in cases from Brazil $(\approx 52 \%)$ [18]. It is far important to announce that the three aforementioned studies were community based. According to the literature, infections with intestinal protozoan infections are commonly asymptomatic [1]. Therefore, high proportions of asymptomatic infections are expected in these studies; on the contrary, in hospital or physician-based study, like our study, where the control group was selected from those patients suffering from diarrhea, asymptomatic protozoan infection was misdiagnosed. This may put an explanation for the high frequency of protozoan infections in the above studies, especially in the control groups compared to our study. Perhaps, variation of the geographical distribution of protozoa, the socioeconomic status of the target population, the demographic characters of participants, and the adopted methodology may be additional explanations for prevalence variations of these protozoan infections among studies.

It has been argued that CRF predispose patients to helminths [11] but this was not the case in our study where no helminths were detected in all participants. Most of our study's participants were urban, middle aged, and using treated bottled water for drinking. These demographic characters may give reasons for the absence of worms' infections that have been broadly recognized by their intimate connection with poverty, remote rural areas, and young ages [19]. Another important reason to be considered is that patients with CRF usually change their health behavior and receive much more health care attention than their peers in populations.

Blastocystis spp. were the most frequently isolated protozoa in the present study. These protozoa were detected more in cases than in controls, consistent with earlier studies $[16,17]$. Higher prevalence rates $(\approx 24 \% ; 34 / 142$ in cases versus $10 \% ; 16 / 150$ in the controls) of Blastocystis spp. infections have been described in the Turkish study [16]. In the Brazilian study, infections have been described in cases $(21 \% ; 18 / 86)$ only [17]. Inconsistent with our finding, Blastocystis spp. have been identified more in controls $(\approx 42 \% ; 36 / 86)$ than in cases $(24.5 \% ; 27 / 110)$, according to Gil et al. [18]. In a cross-sectional Iranian study, Blastocystis spp., with prevalence rate of $13.6 \%$ $(12 / 88)$, have been reported in dialysis patients [20]. Lower prevalence rates $(0.3-14 \%)$ have been previously reported for this intestinal protozoan in Saudi normal populations [2125].

Considering the opportunistic enteric protozoa, neither C. belli nor Microsporidia spp. were identified in our study. Except for a recently published case report for C. belli infection overwhelming a patient with eosinophilic gastroenteritis [26], little has been published for the frequency of these protozoa in Saudi Arabia. Low frequency of Microsporidium spp. infections $(2 \% ; 3 / 142)$ has been reported in Turkish patients with CRF [16]. High prevalence rate of Cryptosporidium spp. was revealed in the present study. While higher rates (11-35\%) have been described in earlier studies 
TABLE 4: The clinical characteristics variations among Cryptosporidium species positive/negative diarrheal cases and controls.

\begin{tabular}{|c|c|c|c|c|c|c|c|c|c|c|}
\hline \multirow{3}{*}{ Clinical character } & \multicolumn{5}{|c|}{ Cryptosporidium species-positive } & \multicolumn{5}{|c|}{ Cryptosporidium species-negative } \\
\hline & \multicolumn{2}{|c|}{ Cases } & \multicolumn{2}{|c|}{ Controls } & \multirow{2}{*}{$P$ value } & \multicolumn{2}{|c|}{ Cases } & \multicolumn{2}{|c|}{ Controls } & \multirow{2}{*}{$P$ value } \\
\hline & $n$ & $\%$ & $n$ & $\%$ & & $n$ & $\%$ & $n$ & $\%$ & \\
\hline \multicolumn{11}{|l|}{ Diarrhea } \\
\hline$<14$ days & 3 & $60 \%$ & 3 & $100 \%$ & \multirow{2}{*}{0.46 (NS) } & 37 & $82.2 \%$ & 47 & $100 \%$ & \multirow{2}{*}{0.002 (Sig) } \\
\hline$>14$ days & 2 & $40 \%$ & 0 & $0 \%$ & & 8 & $17.8 \%$ & 0 & $0 \%$ & \\
\hline \multicolumn{11}{|l|}{ Mucous/blood } \\
\hline Yes & 0 & $0 \%$ & 0 & $0 \%$ & \multirow{2}{*}{ - } & 6 & $13.3 \%$ & 3 & $6.4 \%$ & \multirow{2}{*}{0.31 (NS) } \\
\hline No & 5 & $100 \%$ & 3 & $100 \%$ & & 39 & $86.7 \%$ & 44 & $93.6 \%$ & \\
\hline \multicolumn{11}{|l|}{ Abdominal pain } \\
\hline Yes & 5 & $100 \%$ & 1 & $33.3 \%$ & \multirow{2}{*}{0.10 (NS) } & 45 & $100 \%$ & 38 & $80.9 \%$ & \multirow{2}{*}{0.003 (Sig) } \\
\hline No & 0 & $0 \%$ & 2 & $66.7 \%$ & & 0 & $0 \%$ & 9 & $19.1 \%$ & \\
\hline \multicolumn{11}{|l|}{ Nausea/vomiting } \\
\hline Yes & 3 & $60 \%$ & 3 & $100 \%$ & \multirow{2}{*}{0.46 (NS) } & 11 & $24.4 \%$ & 14 & $29.8 \%$ & \multirow{2}{*}{0.64 (NS) } \\
\hline No & 2 & $40 \%$ & 0 & $0 \%$ & & 34 & $75.6 \%$ & 33 & $70.2 \%$ & \\
\hline \multicolumn{11}{|l|}{ Bloating/flatulence } \\
\hline Yes & 4 & $80 \%$ & 3 & $100 \%$ & \multirow{2}{*}{1.0 (NS) } & 30 & $66.7 \%$ & 13 & $27.7 \%$ & \multirow{2}{*}{0.000 (HS) } \\
\hline No & 1 & $20 \%$ & 0 & $0 \%$ & & 15 & $33.3 \%$ & 34 & $72.3 \%$ & \\
\hline \multicolumn{11}{|l|}{ Fatigue } \\
\hline Yes & 5 & $100 \%$ & 3 & $100 \%$ & \multirow{2}{*}{-} & 45 & $100 \%$ & 15 & $31.9 \%$ & \multirow{2}{*}{0.000 (HS) } \\
\hline No & 0 & $0 \%$ & 0 & $0 \%$ & & 0 & $0 \%$ & 32 & $68.1 \%$ & \\
\hline \multicolumn{11}{|l|}{ Loss of weight } \\
\hline Yes & 1 & $20 \%$ & 1 & $33.3 \%$ & \multirow{3}{*}{$1.0(\mathrm{NS})$} & 5 & $11.1 \%$ & 5 & $10.6 \%$ & \multirow{3}{*}{1.0 (NS) } \\
\hline No & 4 & $80 \%$ & 2 & $66.7 \%$ & & 40 & $88.9 \%$ & 42 & $89.4 \%$ & \\
\hline Total & 5 & $100 \%$ & 3 & $100 \%$ & & 45 & $100 \%$ & 47 & $100 \%$ & \\
\hline
\end{tabular}

$n$ : number, NS: nonsignificant, Sig: significant, and HS: highly significant. $P<0.005$.

TABLE 5: The clinical characteristics variations among Giardia duodenalis positive/negative diarrheal cases and controls.

\begin{tabular}{|c|c|c|c|c|c|c|c|c|c|c|}
\hline \multirow{3}{*}{ Clinical characters } & \multicolumn{5}{|c|}{ Giardia duodenalis-positive } & \multicolumn{5}{|c|}{ Giardia duodenalis-negative } \\
\hline & \multicolumn{2}{|c|}{ Cases } & \multicolumn{2}{|c|}{ Controls } & \multirow{2}{*}{$P$ value } & \multicolumn{2}{|c|}{ Cases } & \multicolumn{2}{|c|}{ Controls } & \multirow{2}{*}{$P$ value } \\
\hline & $n$ & $\%$ & $n$ & $\%$ & & $n$ & $\%$ & $n$ & $\%$ & \\
\hline \multicolumn{11}{|l|}{ Diarrhea } \\
\hline$<14$ days & 3 & $60 \%$ & 6 & $100 \%$ & \multirow{2}{*}{0.18 (NS) } & 37 & $82.2 \%$ & 44 & $100 \%$ & \multirow{2}{*}{0.006 (Sig) } \\
\hline$>14$ days & 2 & $40 \%$ & 0 & $0 \%$ & & 8 & $17.8 \%$ & 0 & $0 \%$ & \\
\hline \multicolumn{11}{|l|}{ Mucous/blood } \\
\hline Yes & 0 & $0 \%$ & 1 & $16.7 \%$ & \multirow{2}{*}{1.0 (NS) } & 6 & $13.3 \%$ & 2 & $4.5 \%$ & \multirow{2}{*}{0.26 (NS) } \\
\hline No & 5 & $100 \%$ & 5 & $83.3 \%$ & & 39 & $86.7 \%$ & 42 & $95.5 \%$ & \\
\hline \multicolumn{11}{|l|}{ Abdominal pain } \\
\hline Yes & 5 & $100 \%$ & 5 & $83.3 \%$ & \multirow{2}{*}{1.0 (NS) } & 45 & $100 \%$ & 34 & $77.3 \%$ & \multirow{2}{*}{0.00 (HS) } \\
\hline No & 0 & $0 \%$ & 1 & $16.7 \%$ & & 0 & $0 \%$ & 10 & $22.7 \%$ & \\
\hline \multicolumn{11}{|l|}{ Nausea/vomiting } \\
\hline Yes & 4 & $80 \%$ & 5 & $83.3 \%$ & \multirow{2}{*}{1.0 (NS) } & 10 & $22.2 \%$ & 12 & $27.3 \%$ & \multirow{2}{*}{0.62 (NS) } \\
\hline No & 1 & $20 \%$ & 1 & $16.7 \%$ & & 35 & $77.8 \%$ & 32 & $72.7 \%$ & \\
\hline \multicolumn{11}{|l|}{ Bloating/flatulence } \\
\hline Yes & 5 & $100 \%$ & 5 & $83.3 \%$ & \multirow{2}{*}{1.0 (NS) } & 29 & $64.4 \%$ & 11 & $25 \%$ & \multirow{2}{*}{0.000 (HS) } \\
\hline No & 0 & $0 \%$ & 1 & $16.7 \%$ & & 16 & $35.6 \%$ & 33 & $75 \%$ & \\
\hline \multicolumn{11}{|l|}{ Fatigue } \\
\hline Yes & 5 & $100 \%$ & 6 & $100 \%$ & \multirow{2}{*}{-} & 45 & $100 \%$ & 12 & $27.3 \%$ & \multirow{2}{*}{0.000 (HS) } \\
\hline No & 0 & $0 \%$ & 0 & $0 \%$ & & 0 & $0 \%$ & 32 & $72.7 \%$ & \\
\hline \multicolumn{11}{|l|}{ Loss of weight } \\
\hline Yes & 0 & $0 \%$ & 1 & $16.7 \%$ & \multirow{3}{*}{1.0 (NS) } & 6 & $13.3 \%$ & 5 & $11.4 \%$ & \multirow{3}{*}{1.0 (NS) } \\
\hline No & 5 & $100 \%$ & 5 & $83.3 \%$ & & 39 & $86.7 \%$ & 39 & $88.6 \%$ & \\
\hline Total & 5 & $100 \%$ & 6 & $100 \%$ & & 45 & $100 \%$ & 44 & $100 \%$ & \\
\hline
\end{tabular}

$n$ : number, NS: nonsignificant, Sig: significant, and HS: highly significant. $P<0.005$. 
[27-29], lower estimates (2-8\%) have been reported in others $[16,30]$. Cryptosporidium spp. infection has been stated with prevalence rate of $2.7-8.1 \%$ in Saudi general populations [23, 25]. Identification of C. cayetanensis in two patients with CRF was interesting. To the best of our knowledge, this protozoan has never been reported in patients with CRF. In Saudi Arabia, C. cayetanensis has been rarely detected even in immunocompromised patients [23].

Giardia duodenalis was detected in considerable number of the study's participants. Infections were identified more in the control group, compatible with an earlier study [18] and incompatible with another [16]. Giardia duodenalis has been commonly detected in Saudi communities. Infection rates of 3-18\% have been reported [12, 22-26]. Moreover, E. histolytica, the tissue-invasive intestinal protozoan, was rarely determined among cases in this study, consistent with a previous study [17] and inconsistent with other [31]. E. histolytica infection has been recognized as a public health threat in Saudi Arabia. Prevalence rates of 6-35\% have been described in Saudi Arabia [13, 21-24]. Interestingly, in a study carried out in Saudi population, an infection rate of $\approx 60 \%$ has been described [25]. In the aforementioned studies, microscopy has been used as a sole technique for diagnosis of amoebiasis. The low sensitivity of microscopy and its incompetence to determine the morphologically identical species may put an explanation for these exaggerated prevalence rates.

Diarrhea was often acute and transient in most of the parasitized patients. Persistent diarrhea was reported by few parasitized cases, consistent with previous report [32]. Persistent diarrhea was described with all the recognized protozoa except $E$. histolytica. Acute dysenteric diarrhea was described in six cases, two of them were found parasitized with Blastocystis spp. concomitantly with G. duodenalis in one patient and with E. histolytica in the other. In addition, acute dysenteric diarrhea was observed in three controls, one of them was found parasitized with E. histolytica. In the presence of coinfection, it is very challenging to attribute patients' symptoms to one protozoan and neglect the other [33]. Perhaps, one may attribute dysentery to E. histolytica being a well-known tissue-invasive parasite [31]. One else may relate the dysenteric diarrhea to Blastocystis infection relying on a previous research $[34,35]$. Any other dysenteric cases that have not been supported by the literature require further investigations.

This is the first study comparing the frequency of intestinal protozoa-associated diarrhea in CRF patients and nonrenal controls from Taif, Saudi Arabia. In this study, special considerations were given to avoid well-known potential confounders while enrolling patients and subsequent selection bias. Another strength of our study was the inclusion of a number of immunoassays in the study's methodology to avoid the low sensitivity of microscopy-based methods in diagnosis of certain parasites such as E. histolytica. Nevertheless, our study was not free from the limitations. Low number of cases was enrolled in this study due to the strict selection criteria adopted. We could not rely on the recent immune status of participants based on doing laboratory tests because many participants were reluctant to give blood samples. Lastly, we did not include tests for intestinal bacteria and viruses that could be concomitantly present with protozoan infection(s), due to time and cost limits of our study. All these limitations plus performing longitudinal study have to be considered in the near future research.

In conclusion, high frequency of intestinal protozoan infections was described in the study's population. Protozoa were detected more in patients with CRF than in diarrheal controls. Further studies are required before attributing diarrhea in patients with CRF to a protozoan detected in patients' feces. Until these studies, we advise screening all diarrheal patients for intestinal protozoa in the study's population, with greater concern that should be given to patients with CRF.

\section{Conflict of Interests}

The authors declare that there is no conflict of interests regarding the publication of this paper.

\section{Acknowledgments}

The authors would like to thank all staff and technicians at the Medical Laboratory Department, College of Applied Medical Sciences, for their support while doing this research. Special thanks go to physicians at the dialysis unit and the outpatient clinics at King Abdulaziz General Hospital for their help and support in recruiting patients in the study.

\section{References}

[1] V. A. Cama and B. A. Mathison, "Infections by intestinal coccidia and Giardia duodenalis," Clinics in Laboratory Medicine, vol. 35, no. 2, pp. 423-444, 2015.

[2] P. R. Torgerson, N. R. de Silva, E. M. Fèvre et al., "The global burden of foodborne parasitic diseases: an update," Trends in Parasitology, vol. 30, no. 1, pp. 20-26, 2014.

[3] S. Baldursson and P. Karanis, "Waterborne transmission of protozoan parasites: review of worldwide outbreaks-an update 2004-2010," Water Research, vol. 45, no. 20, pp. 6603-6614, 2011.

[4] M. Agholi, G. R. Hatam, and M. H. Motazedian, "HIV/AIDSassociated opportunistic protozoal diarrhea," AIDS Research and Human Retroviruses, vol. 29, no. 1, pp. 35-41, 2013.

[5] B. G. Sard, R. T. Navarro, and J. G. E. Sanchis, "Nonpathogenic intestinal amoebae: a clinical-analytical overview," Enfermedades Infecciosas y Microbiología Clínica, vol. 29, no. 3, pp. 20-28, 2011.

[6] S. Basak, M. N. Rajurkar, and S. K. Mallick, "Detection of Blastocystis hominis: a controversial human pathogen," Parasitology Research, vol. 113, no. 1, pp. 261-265, 2014.

[7] L. A. Marcos and E. Gotuzzo, "Intestinal protozoan infections in the immunocompromised host," Current Opinion in Infectious Diseases, vol. 26, no. 4, pp. 295-301, 2013.

[8] G. Eknoyan and N. W. Levin, "K/DOQI clinical practice guidelines for chronic kidney disease: evaluation, classification, and stratification-foreword," American Journal of Kidney Diseases, vol. 39, no. 2, pp. S14-S266, 2002.

[9] H.-J. Anders, K. Andersen, and B. Stecher, "The intestinal microbiota, a leaky gut, and abnormal immunity in kidney disease," Kidney International, vol. 83, no. 6, pp. 1010-1016, 2013.

[10] G. Cohen and W. H. Hörl, "Immune dysfunction in uremia-an update," Toxins, vol. 4, no. 11, pp. 962-990, 2012. 
[11] R. M. Manesh, A. H. Safa, S. M. Sharafi et al., "Parasites and chronic renal failure," Journal of Renal Injury Prevention, vol. 3 , no. 4, pp. 87-90, 2014.

[12] F. A. Al-Braiken, "Is intestinal parasitic infection still a public health concern among Saudi children?" Saudi Medical Journal, vol. 29, no. 11, pp. 1630-1635, 2008.

[13] A. A. Al-Sayyari and F. A. Shaheen, "End stage chronic kidney disease in Saudi Arabia. A rapidly changing scene," Saudi Medical Journal, vol. 32, no. 4, pp. 339-346, 2011.

[14] I. H. McHardy, M. Wu, R. Shimizu-Cohen, M. Roger Couturier, and R. M. Humphries, "Detection of intestinal protozoa in the clinical laboratory," Journal of Clinical Microbiology, vol. 52, no. 3, pp. 712-720, 2014.

[15] L. S. Garcia, Practical Guide to Diagnostic Parasitology, ASM Press, Washington, DC, USA, 2nd edition, 2009.

[16] G. Karadag, G. S. Tamer, and E. Dervisoglu, "Investigation of intestinal parasites in dialysis patients," Saudi Medical Journal, vol. 34, no. 7, pp. 714-718, 2013.

[17] R. A. Kulik, D. L. M. Falavigna, L. Nishi, and S. M. Araujo, "Blastocystis sp. and other intestinal parasites in hemodialysis patients," Brazilian Journal of Infectious Diseases, vol. 12, no. 4, pp. 338-341, 2008.

[18] F. F. Gil, M. J. Barros, N. A. Macedo et al., "Prevalence of intestinal parasitism and associated symptomatology among hemodialysis patients," Revista do Instituto de Medicina Tropical de São Paulo, vol. 55, no. 2, pp. 69-74, 2013.

[19] J. Utzinger, "A research and development agenda for the control and elimination of human helminthiases," PLoS Neglected Tropical Diseases, vol. 6, no. 4, article e1646, 2012.

[20] A. Barazesh, M. Fouladvand, R. Tahmasebi, A. Heydari, and J. Fallahi, "The prevalence of intestinal parasites in hemodialysis patients in Bushehr, Iran," Hemodialysis International, vol. 19, no. 3, pp. 447-451, 2015.

[21] A. Imam, A. Altayyar, E. Eltayeb et al., "Frequency and seasonality of intestinal parasitism in Qassim region, Saudi Arabia," Pakistan Journal of Medical Sciences, vol. 28, pp. 913-916, 2012.

[22] Z. A. Khan and I. S. Alkhalife, "Prevalence of Blastocystis hominis among 'healthy' food handlers in Dammam, Saudi Arabia," Journal of the Egyptian Society of Parasitology, vol. 35, no. 2, pp. 395-401, 2005.

[23] W. A. I. Al-Megrin, "Intestinal parasites infection among immunocompromised patients in Riyadh, Saudi Arabia," Pakistan Journal of Biological Sciences, vol. 13, no. 8, pp. 390-394, 2010.

[24] H. I. Al-Mohammed, T. T. Amin, E. Aboulmagd, H. R. Hablus, and B. O. Zaza, "Prevalence of intestinal parasitic infections and its relationship with socio-demographics and hygienic habits among male primary schoolchildren in Al-Ahsa, Saudi Arabia," Asian Pacific Journal of Tropical Medicine, vol. 3, no. 11, pp. 906912, 2010.

[25] S. A. Al-Harthi and M. B. Jamjoom, "Enteroparasitic occurrence in stools from residents in Southwestern region of Saudi Arabia before and during Umrah season," Saudi Medical Journal, vol. 28, no. 3, pp. 386-389, 2007.

[26] U. Navaneethan, P. G. Venkatesh, E. Downs-Kelly, and B. Shen, "Isospora belli superinfection in a patient with eosinophilic gastroenteritis-a diagnostic challenge," Journal of Crohn's and Colitis, vol. 6, no. 2, pp. 236-239, 2012.

[27] P. P. Chieffi, Y. A. Sens, M. A. Paschoalotti, L. A. Miorin, H. G. Silva, and P. Jabur, "Infection by Cryptosporidium parvum in renal patients submitted to renal transplant or hemodialysis,"
Revista da Sociedade Brasileira de Medicina Tropical, vol. 31, no. 4, pp. 333-337, 1998.

[28] R. Baqai, S. Anwar, and S. U. Kazmi, "Detection of Cryptosporidium in immunosuppressed patients," Journal of Ayub Medical College, Abbottabad, vol. 17, no. 3, pp. 38-40, 2005.

[29] K. H. Hazrati Tappeh, M. J. Gharavi, K. Makhdoumi, M. Rahbar, and A. Taghizadeh, "Prevalence of Cryptosporidium spp. infection in renal transplant and hemodialysis patients," Iranian Journal of Public Health, vol. 35, no. 3, pp. 54-57, 2006.

[30] N. Turkcapar, S. Kutlay, G. Nergizoglu, T. Atli, and N. Duman, "Prevalence of Cryptosporidium infection in hemodialysis patients," Nephron, vol. 90, no. 3, pp. 344-346, 2002.

[31] S. R. Ferreira-Filho, F. C. A. da Costa Braga, D. M. de Sa et al., "Entamoeba histolytica/Entamoeba dispar infection in chronic hemodialysis patients," Saudi Journal of Kidney Diseases and Transplantation, vol. 22, no. 2, pp. 237-244, 2011.

[32] S. W. Pawlowski, C. A. Warren, and R. Guerrant, "Diagnosis and treatment of acute or persistent diarrhea," Gastroenterology, vol. 136, no. 6, pp. 1874-1886, 2009.

[33] J. Lello, S. Knopp, K. A. Mohammed, I. S. Khamis, J. Utzinger, and M. E. Viney, "The relative contribution of co-infection to focal infection risk in children," Proceedings of the Royal Society of London B: Biological Sciences, vol. 280, no. 1754, Article ID 20122813, 2013.

[34] W.-P. Tai, P.-J. Hu, J. Wu, and X.-C. Lin, "Six ulcerative colitis patients with refractory symptoms co-infective with Blastocystis hominis in China," Parasitology Research, vol. 108, no. 5, pp. 1207-1210, 2011.

[35] N. Andiran, Z. C. Acikgoz, S. Turkay, and F. Andiran, “Blastocystis hominis-an emerging and imitating cause of acute abdomen in children," Journal of Pediatric Surgery, vol. 41, no. 8, pp. 14891491, 2006. 


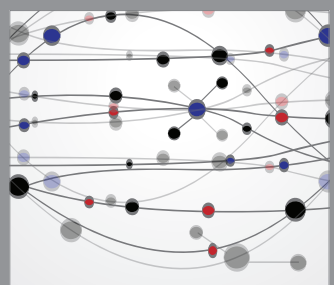

The Scientific World Journal
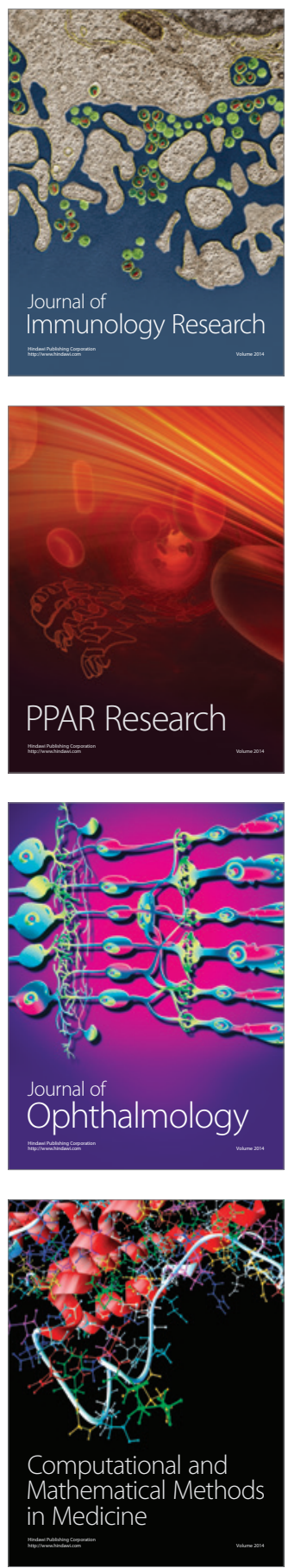

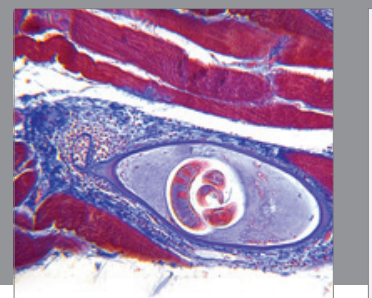

Gastroenterology

Research and Practice
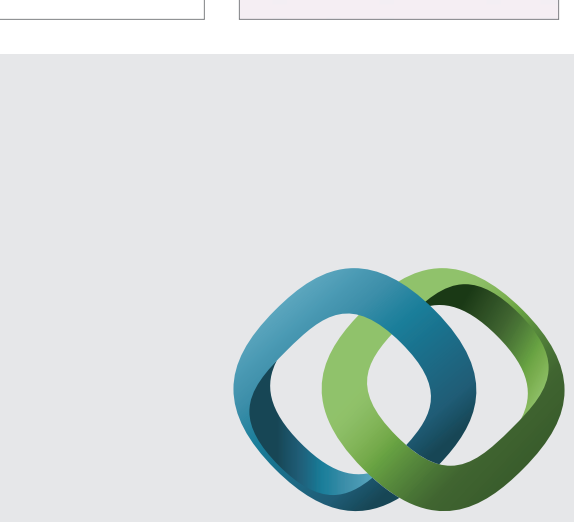

\section{Hindawi}

Submit your manuscripts at

http://www.hindawi.com
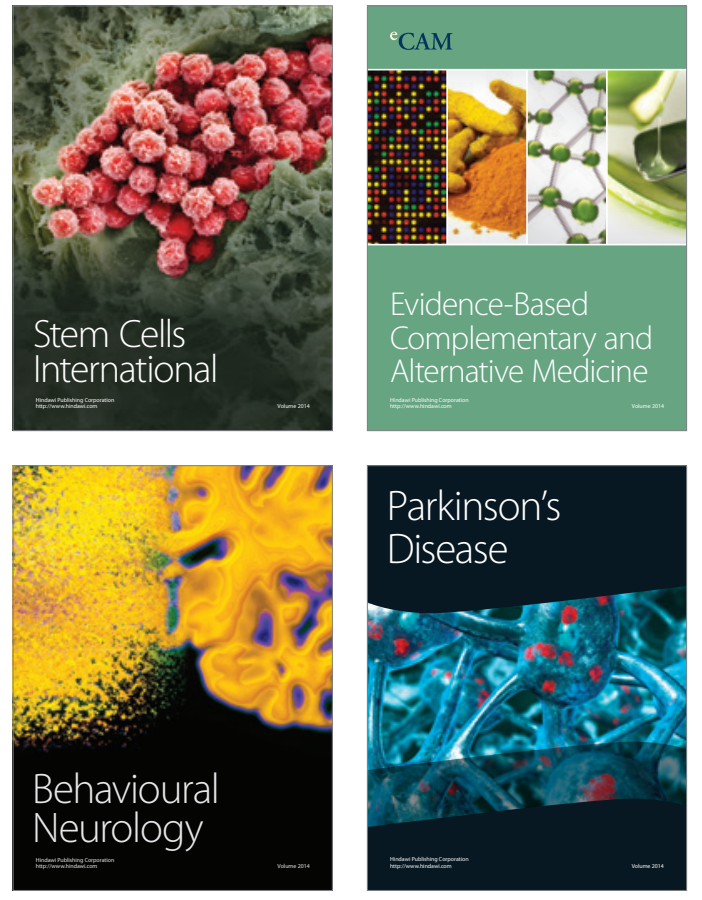
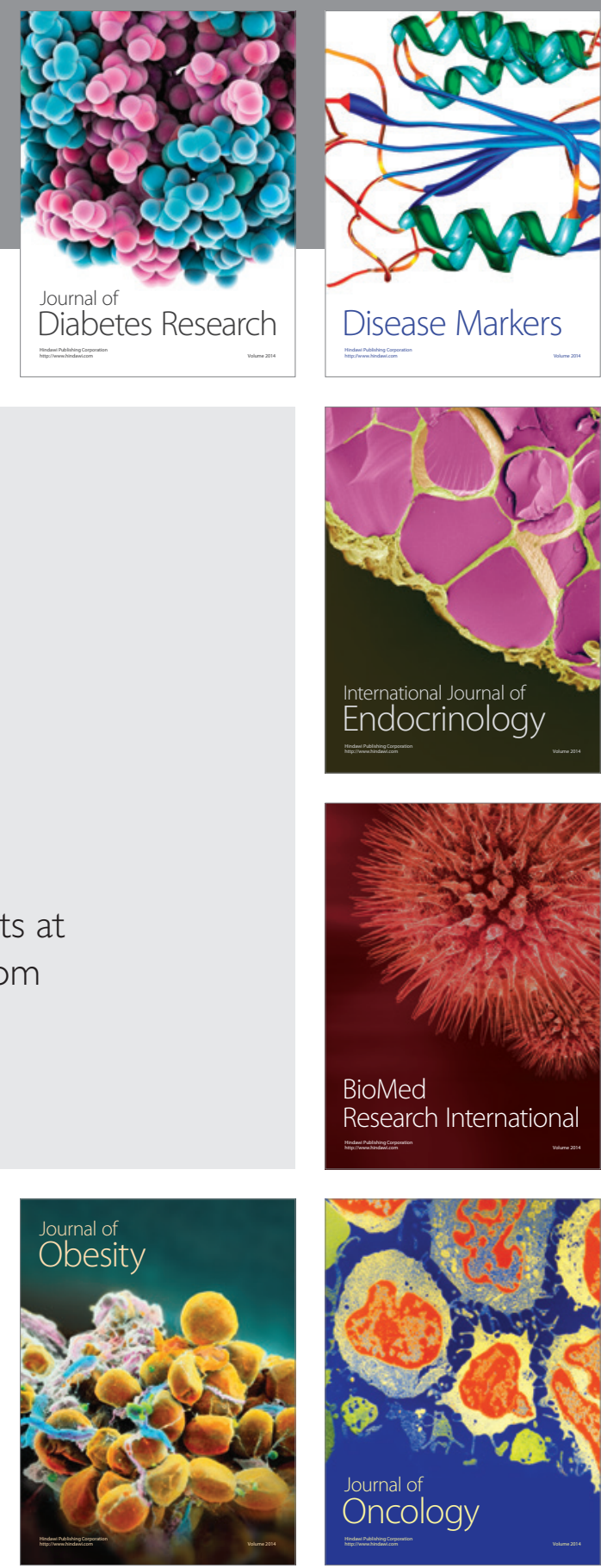

Disease Markers
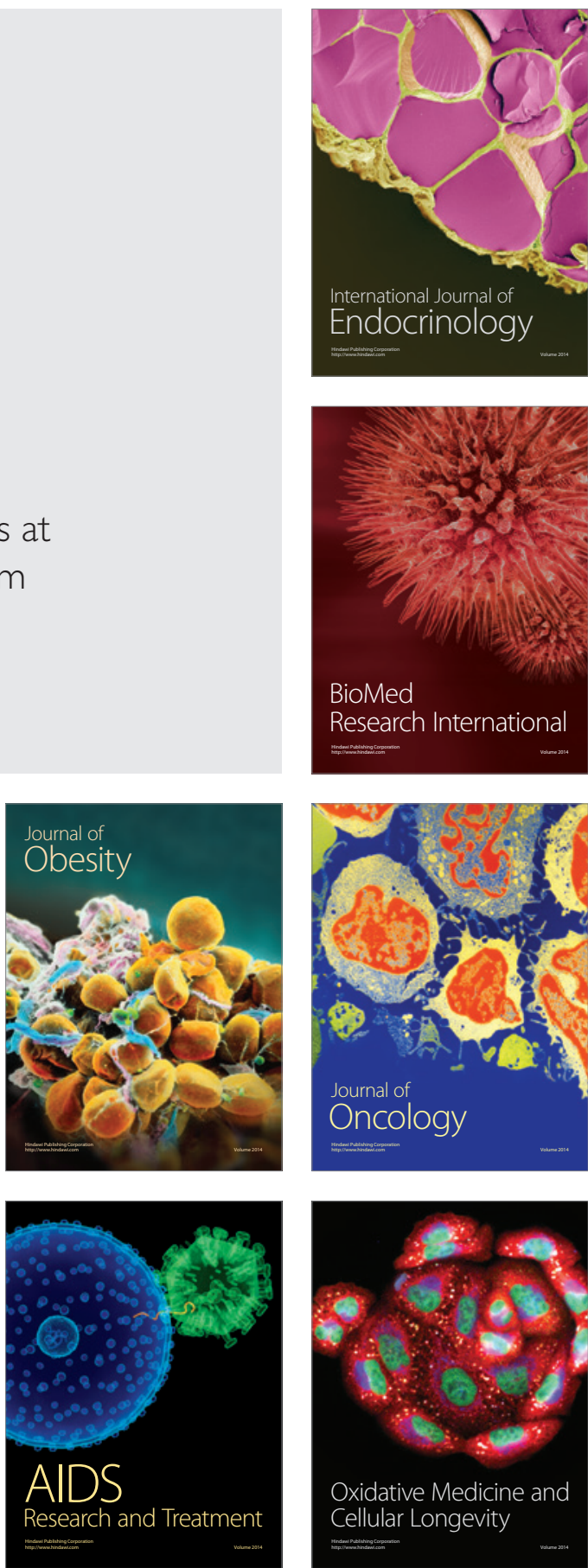